\title{
Testing of numerical simulation technique of concentrated suspensions agitation
}

\author{
Vladimir Zhigarev ${ }^{1, *}$, Andrey Minakov ${ }^{1,2}$, Dmitry Platonov ${ }^{1,2}$, Dmitry Dekterev ${ }^{1,2}$ and \\ Maxim Pryazhnikov ${ }^{1,2}$ \\ ${ }^{1}$ Siberian Federal University, 79 Svobodny pr., Krasnoyarsk, 660041, Russia \\ ${ }^{2}$ Institute of Thermophysics SB RAS, 1 Acad. Lavrentiev pr., Novosibirsk, 630090, Russia
}

\begin{abstract}
The paper presents testing results of the numerical technique to simulate the agitation process of concentrated suspensions. The simulating technique is based on Eulerian two-fluid model of granular media, k-w SST URANS turbulence model, and sliding mesh approach. The results obtained show a good qualitative and quantitative agreement between calculation and experiment in terms of the shape and location of the phase interface and the distribution of the solid phase along the height.
\end{abstract}

\section{Introduction}

Solid-liquid systems occur in a wide range of industrial applications such as mineral processing, precipitation, biochemical crystallization processes, and drilling of the wells. A considerable amount of work has been done to investigate the influence of hydrodynamics and mixing of solid suspensions using impellers. Mixing is the one of the most common ways to enhance many engineering processes [1]. Apparatuses with mixing devices are used in processes such as crystallization, absorption, extraction, homogeneous and heterogeneous chemical reactions, etc. The most efficient way of mixing is creation of swirling flow inside the reactor volume. Swirling flow can be created using a variety of mixing devices (agitators). Apparatuses with mechanical impellers are preferably used in processes with prevailing liquid phase. Up to now, $80 \%$ of application processes consist of blending, and at the same time $50 \%$ of them deal with solid-liquid suspensions [2]. These operations require employment of axial high speed impellers, because their flow characteristics significantly affect both off-bottom suspension [3] and the rate of homogenization of liquid phase in agitated charges [4].

\section{Testing of numerical methods}

The objective of the present work was testing the computational technique to simulate the suspensions agitation processes by means of devices with a swirling flow (agitators). Calculation of suspension agitation process is implemented using a hydrodynamic approach based on the solution of Reynolds averaged Navier-Stokes equations with a two-fluid Eulerian model for granular media [5].

\footnotetext{
*Corresponding author: zhigarev.vladimir@yandex.ru
} 
The agitation of silicon carbide particles suspension in a cylindrical container was considered as the first task to test the computational technique. A detailed description of this experiment can be found in [6].

The computational domain was a cylindrical volume with a diameter of $240 \mathrm{~mm}$ and a height of $225 \mathrm{~mm}$. The inner wall of the cylinder had 4 ribs with the dimensions of $25 \times 10 \times 225 \mathrm{~mm}$. Agitation of the pulp in the cylinder was carried out by installed flat fourblade impeller with the blade angle of 45 degrees. The impeller had the following dimensions: diameter $-105 \mathrm{~mm}$, blade width $-14 \mathrm{~mm}$, and blade thickness $-2 \mathrm{~mm}$. The rotation speed of the impeller was $300 \mathrm{rpm}$. Photograph of the experimental setup [6] is shown in figure 1 . The experiment was conducted for volumetric concentration of silicon carbide particles equal to $5 \%$, particle size was equal to $500 \mu \mathrm{m}$. Water was used as an actuating medium. As a result of the experiments we measured the distribution of the bulk concentration of particles along the height of the cylinder.

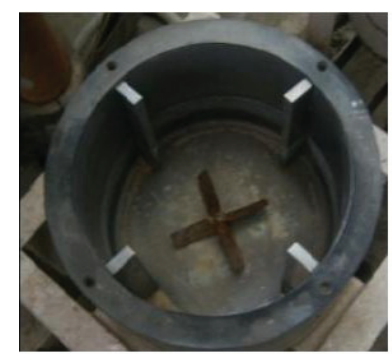

Fig. 1. Photograph of the experimental setup taken from [6].

Place the figure as close as possible after the point where it is first referenced in the text. If there is a large number of figures and tables it might be necessary to place some before their text citation.

In calculations we used unstructured mesh consisting of 950000 cells with refinement in their number towards actuator walls. The impeller rotation was simulated using the "frozen rotor" approach. The problem was solved for spatial stationary conditions. For simulation we used k-w SST (RANS) turbulence model. For simulation of the pulp agitation we used Eulerian two-fluid model of granular media. The coefficient of hydraulic resistance of the particles was set according to the Syamlal-Obrien model, the lifting force was simulated according to the Saffman-Mei lift model, turbulent dispersion was simulated employing Simonin model, granular viscosity was calculated according to Gidaspow, while stresses in the solid phase - by the model of Lun at al.

Figure 2 shows the distribution of the particles volume fraction over the walls of the cylindrical area. As is obvious, the distribution of particles is rather uneven. Due to centrifugal force, particles are concentrated at the cylinder walls and in the corners of the ribs.
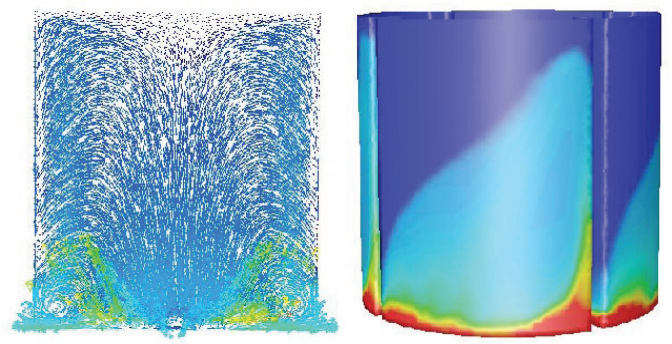

Fig. 2. The velocity field in the central plane (left) and the distribution of the particles volume fraction over the walls (right). 
The results of quantitative comparison with experiment are shown in Fig. 3. The figure shows the distribution of particles volume fraction over the cylinder height along the line lying on the inner wall of the cylinder midway between the ribs. The diagram shows the results obtained using Eulerian two-fluid model without taking into account turbulent diffusion, though considering the model of Simonin [7]. As is obvious, not taking into account turbulent dispersion leads to a significant underestimation of the particles lifting height. This parameter is very important in the chemical heterophase devices. The Simonin model of turbulent dispersion gives satisfactory agreement with experiment on the particle concentration distribution over the height of the computational domain.

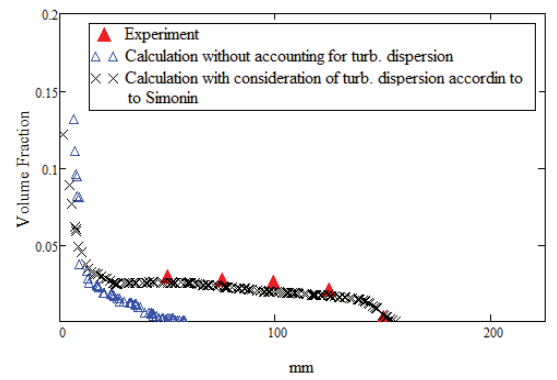

Fig. 3. The distribution of the volume fraction of solid phase over the height of the computational domain.

\section{The experimental results and numerical calculation}

A special experimental setup was designed for a more detailed study of the pulp agitation process. Photograph of the setup is shown in Fig. 4. The experimental setup is a glass cylindrical container served as a stirred tank with a diameter of $20 \mathrm{~cm}$ and a height of $40 \mathrm{~cm}$.

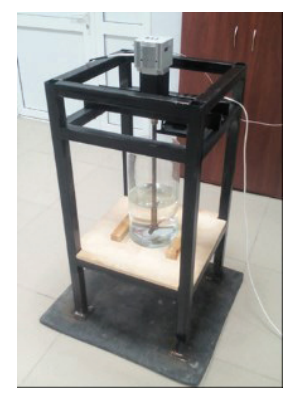

Fig. 4. Photograph of the experimental setup.

The rotation of the agitator was carried out using a servomotor. The rotation speed of the agitator was controlled within the range from 0 to $3000 \mathrm{rpm}$. The distribution of particles in a fluid in the course of the experiment was recorded using high-speed camera. For testing mathematical model we conducted simulation of agitation process of $500 \mu \mathrm{m}$ sand particles at a volume concentration of $10 \%$. The agitation of two-phase medium was carried out by four-blade flat impeller with the blade angle of 45 degrees. The agitator had the following dimensions: diameter $-80 \mathrm{~mm}$, blade width $-10 \mathrm{~mm}$, and blade thickness $2 \mathrm{~mm}$. The agitator was mounted at a height of $20 \mathrm{~mm}$ from the bottom of the container and in this case had no guide ribs. The rotation speed of the agitator was varied from 100 to $800 \mathrm{rpm}$. In our calculations we used unstructured mesh consisting of 1250000 cells, with refinement towards the agitator walls. The simulation was performed under unsteady conditions. In our simulation we used k-w SST (URANS) turbulence model. For simulation 
of the pulp agitation we used Eulerian two-fluid model with the sub-models described above. Simulation of the agitator rotation was carried out by a "sliding mesh" model. Examples of comparison are presented in Figs. 5.

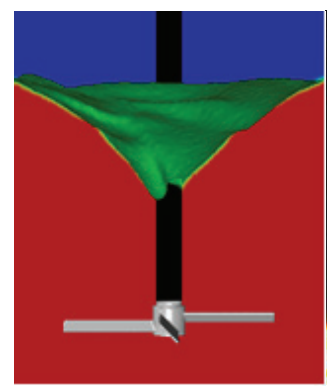

a

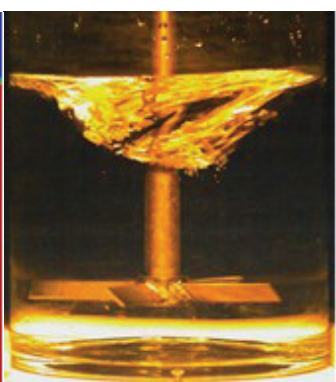

$\mathrm{b}$

Fig. 5. A comparison of the volume fraction of the liquid with the experimental image at a rotation speed of $200 \mathrm{rpm}$ without solid phase. a) Numerical simulation, b) experiment.

First, the experiment was carried out at zero concentration of particles. Figure $6 \mathrm{~b}$ shows a photograph of such an experiment. As can be seen, the absence of the solid phase and guide ribs leads to the appearance of a large funnel. For the simulation of wave generation on a free surface, VOF method was used in simulation [7-9]. The presence of a funnel during the agitation process has negative effect, since at high rotation speeds the air can reach the agitator blades and mixing efficiency will be sharply reduced. This effect is called "flooding" of agitator. As can be seen from Fig. 6a, the calculated shape of the free surface very well corresponds to the funnel observed experimentally.

Next, we conducted a series of experiments with solid particles. The results of the experiments and simulations are shown in Figs.6-7. As is obvious, in the first case $(200 \mathrm{rpm})$ no pulp agitation was observed. The phase interface remains almost flat. At the agitator rotation speed of $300 \mathrm{rpm}$ there is strong enough wave generation on the free surface of the fluid and the interface between liquid and solid phase. In this mode, the pulp is moving wavily (see Fig.7). However, agitation in this mode cannot be called effective either. Homogeneous agitation of the particles can only be achieved at the rotation speeds of the agitator exceeding $600 \mathrm{rpm}$. Qualitative and quantitative comparison of calculation and experiment on the lift height of the particles has shown that for all cases the calculation very well describes the behavior of the pulp in the experiment. The results are shown in Fig. 5.

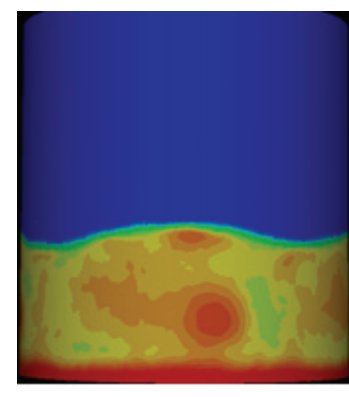

a

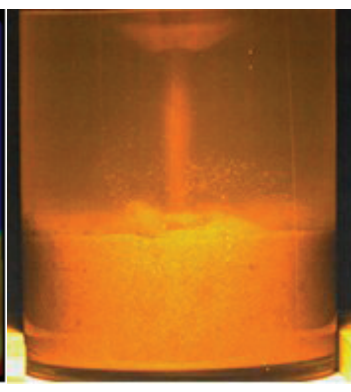

b

Fig. 6. A comparison of the particles volume fraction at the wall with experiment at rotation speed of $200 \mathrm{rpm}$. a) Numerical simulation, b) experiment. 


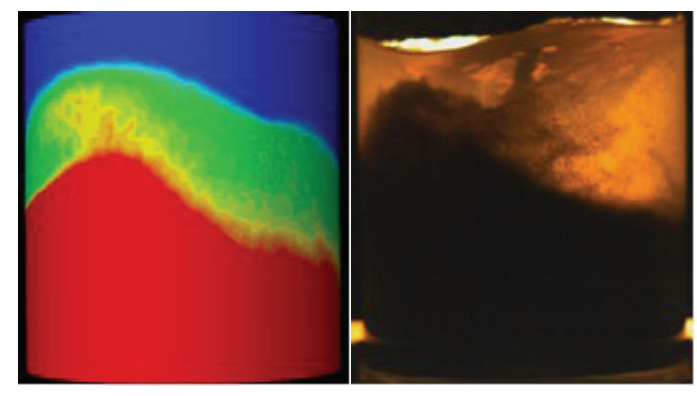

a

b

Fig. 7. A comparison of the particles volume fraction at the wall with experiment at rotation speed of $300 \mathrm{rpm}$. a) Numerical simulation, b) experiment.

\section{Conclusion}

We have conducted a review and analysis of up-to-date types and methods of agitators for chemical heterogeneous processes. A model setup was designed and the experimental study of the mixing process of the $500 \mu \mathrm{m}$ sand particles suspension was carried out. The pulp mixing efficiency at various rotation speeds of the agitator was determined by means of a high-speed camera. The mathematical model and numerical technique was developed to simulate suspension agitation process. The developed numerical technique was tested by comparison of calculations with the own experimental data as well as the data obtained by other authors. Good qualitative and quantitative agreement between calculation and experimental data was achieved in terms of the shape and location of the phase interface and the distribution of the solid phase over the reactor height. In the future we plan to perform a comparative analysis of the mixing efficiency for the agitators with different deign shapes.

The reported study was funded by Russian Foundation for Basic Research, Government of Krasnoyarsk Territory, Krasnoyarsk Region Science and Technology Support Fund to the research project № 16-41-243034.

\section{References}

1. Starback, P. Tausk. L., Chimizdat,(1963).

2. I. For̆t, P. Seichter, L. Pešl, F. Rieger, T. Jirout, Chem. Process Eng. Vol.34 (2013).

3. J. WU, L. PULLUM, J. AICHE, Vol.46 (2000)

4. I. FOŘT, Vol. 3, Academic Press,New York, (1986).

5. ANSYS FLUENT Tutorial Guide. Validation.

6. Lalit Upadhayay, Dissertation PhD (2010)

7. C.W Hirt, B.D Nichols. J. Comput.Phys. Vol. 39 (1981).

8. D.V. Guzei, A.V. Minakov, M.I. Pryazhnikov, A.A. Dekterev. THERMOPHYS AEROMECH+ Vol.22 (2015)

9. A.V. Minakov. COMP MATH MATH PHYS+. Vol. 54 (2014) 